\title{
Atomistic study of the structural and electronic properties of a-Si:H/c-Si interfaces
}

\author{
Iván Santos $^{1, *}$, Marco Cazzaniga $^{2,3}$, Giovanni Onida ${ }^{2,3}$, Luciano \\ Colombo $^{4}$ \\ ${ }^{1}$ Departamento de Electricidad y Electrónica, Universidad de Valladolid, E.T.S.I. de \\ Telecomunicación, Paseo Belén 15, 47011 Valladolid, Spain \\ 2 Dipartimento di Fisica, Università degli Studi di Milano, Milano, Italy \\ ${ }^{3}$ European Theoretical Spectroscopy Facility (ETSF), Italy \\ ${ }^{4}$ Dipartimento di Fisica, Università degli Studi di Cagliari, Cittadella Universitaria, \\ I-09042 Monserrato (CA), Italy \\ E-mail: *ivasan@tel.uva.es
}

\begin{abstract}
We investigate the structural and electronic properties of the interface between hydrogenated amorphous silicon $(a-\mathrm{Si}: \mathrm{H})$ and crystalline silicon $(c-\mathrm{Si})$ by combining tight-binding molecular dynamics and DFT ab inito electrionic stucture calculations. We focus on the $c-\mathrm{Si}(100)(1 \times 1) / a-\mathrm{Si}: \mathrm{H}, \quad c-\mathrm{Si}(100)(2 \times 1) / a-\mathrm{Si}: \mathrm{H}$ and the $c$-Si(111)/a-Si:H interfaces due to their technological relevance. The analysis of atomic rearrangements induced at the interface by the interaction between $\mathrm{H}$ and $\mathrm{Si}$ allowed us to identify the relevant steps that lead to the transformation from $c$ $\mathrm{Si}(100)(1 \times 1) / a$-Si:H to $c$ - $\mathrm{Si}(100)(2 \times 1) / a$-Si:H. The interface electronic structure is found to be characterized by spatially localized mid-gap states. Through them we have identified the relevant atomic structures responsible for the interface defect states, namely: dangling-bonds, $\mathrm{H}$ bridges, and strained bonds. Our analysis contributes to a better understanding of the role of such defects in $c$-Si $/ a$-Si:H interfaces
\end{abstract}

PACS numbers: 07.05.Tp,68.35.-p,68.35.bg,68.35.bj,73.20.At

Keywords: amorphous-crystalline silicon interface, interface defect states, charge trapping, atomistic simulations

Submitted to: $J P C M$ 


\section{Introduction}

Heterojunctions between crystalline $\mathrm{Si}$ and hydrogenated amorphous $\mathrm{Si}(c-\mathrm{Si} / a-\mathrm{Si}: \mathrm{H})$ have been used for fabricating high-efficiency solar cells, with efficiencies over $20 \%[1,2]$. Further improvements of the efficiency can be achieved, for example, by increasing the open-circuit voltage in the solar cell, which implies a reduction of the carrier recombination losses at the $c$-Si/ $a-\mathrm{Si}: \mathrm{H}$ interface [3]. For that purpose, fabrication processes of solar cells should produce good quality $c$-Si $/ a$-Si:H interfaces with large minority carrier lifetimes at this region, and therefore a low interface defect density.

In order to seek these optimum fabrication processes, a number of different studies have analyzed how the defect density at $c-\mathrm{Si} / a-\mathrm{Si}: \mathrm{H}$ interfaces is affected by annealing treatments [4], the $\mathrm{H}$ content [5] and the doping [6] of the $a$-Si:H layer, or even by light illumination $[7,8]$. Experimental facts found in these studies are explained in terms of a variety of atomic phenomena occurring at the interface vicinity. It is widely assumed that the interface defect density is influenced by the local atomic structure at the interface. The defect density can be reduced with annealing treatments [4] or by passivation of the dangling bonds (i.e. Si atoms with three bonds rather than the four bonds of its natural coordination) at the interface with $\mathrm{H}$ atoms [5]. Nevertheless, the interface defect density is doping-dependent [6], and it can be increased by light illumination $[7,8]$. Such a light-induced increment presents a strong dependence on the orientation of the $c$-Si substrate [8]. Explanations of experimental facts are mainly based on the generation/recombination of dangling bonds and models based on the role of this defect have been developed [9], but there are some open questions and experimental observations that cannot be completely explained. At this point, atomistic simulations can help to identify the relevant defects and phenomena occurring at the interface, providing useful information to improve the existing models.

In this work we have combined different atomistic simulations to perform a structural and electronic characterization of $c$-Si/a-Si:H interfaces for different orientations and terminations of the $c$-Si substrate. We have focused our study on two aspects: we have analyzed how $\mathrm{H}$ atoms interact at the $c$-Si/ $a$-Si:H interface, and we have identified the relevant interface defect states, correlating the latter with the particular atomic structure that induce them.

\section{Simulation details}

We used tight-binding (TB) molecular dynamics simulations within an $s p^{3}$ orthogonal tight-binding approach [10] to generate the atomic models of the $c$-Si/ $a$-Si:H interfaces. We employed a Hamiltonian where $\mathrm{Si}-\mathrm{Si}$ interactions are parametrized according to the model proposed by Goodwin, Skinner, and Pettifor (GSP) [11], that provides an adequate description of defects in $c$-Si $[12,13,14]$, and of the structural properties of $a$-Si $[15,16]$. The Si-H parametrization has been presented in Ref. [17], while the H-H parametrization is discussed in Ref. [18]. The resulting hamiltonian for the Si-H system 


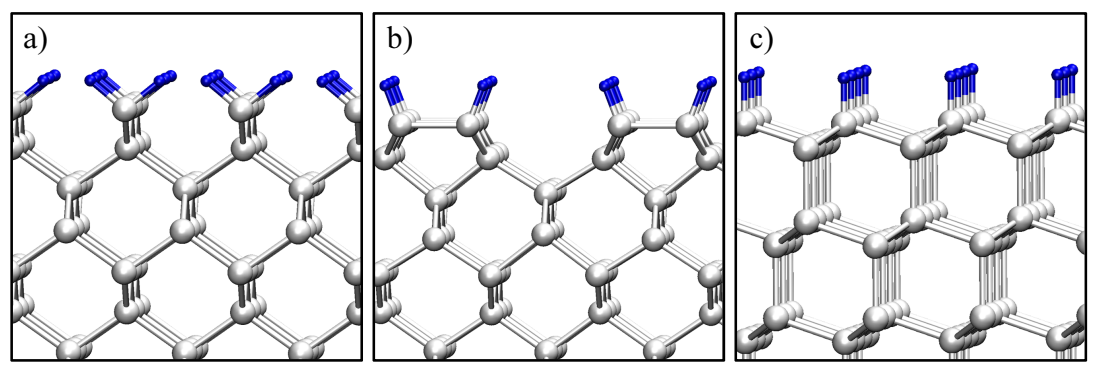

Figure 1. (Color online) H passivation of the different $c$-Si terminations considered in the present study: (a) $c$ - $\mathrm{Si}(100) 1 \times 1$, (b) $c$ - $\mathrm{Si}(100) 2 \times 1$, and (c) $c$-Si(111). Si atoms are represented by open symbols (white atoms), $\mathrm{H}$ atoms are represented by small filled symbols (blue spheres).

\begin{tabular}{lccccccc}
\hline & $L_{x}(\mathrm{~nm})$ & $L_{y}(\mathrm{~nm})$ & $L_{z}(\mathrm{~nm})$ & $N_{S i}$ & $N_{H}$ & $\rho_{H}^{\text {surf. }}\left(\mathrm{nm}^{-2}\right)$ & $N_{\text {conf. }}$ \\
\hline$c$-Si(100 $)(1 \times 1) / a-\mathrm{Si}: \mathrm{H}$ & & & & & & 13.9 & 4 \\
$\quad$ Crystalline side & 1.66 & 1.14 & 1.52 & 144 & 48 & & \\
Amorphous side & 1.62 & 1.14 & 1.52 & 144 & 15 & & \\
Total & 3.28 & 1.14 & 1.52 & 288 & 63 & & \\
\hline$c$-Si(100) $(2 \times 1) / a-\mathrm{Si}: \mathrm{H}$ & & & & & & 6.9 & \\
$\quad$ Crystalline side & 1.72 & 1.14 & 1.52 & 144 & 24 & & \\
Amorphous side & 1.62 & 1.14 & 1.52 & 144 & 15 & & \\
Total & 3.34 & 1.14 & 1.52 & 288 & 39 & & \\
\hline$c$-Si(111)/a-Si:H & & & & & & 8.0 & \\
Crystalline side & 1.92 & 1.32 & 1.52 & 192 & 32 & & \\
Amorphous side & 1.87 & 1.32 & 1.52 & 192 & 20 & & \\
Total & 3.79 & 1.32 & 1.52 & 384 & 52 & & \\
\hline
\end{tabular}

Table 1. Summary of the structural features of the simulation cells used: size of the $c$-Si and $a$-Si:H slabs for the DFT simulations, number of $\mathrm{Si}$ and $\mathrm{H}$ atoms, surface density of $\mathrm{H}$ atoms $\left(\rho_{H}^{\text {surf. }}\right)$, and number $\left(N_{\text {conf. }}\right)$ of different configurations considered for each $c-\mathrm{Si} / a-\mathrm{Si}: \mathrm{H}$ interface.

has been successfully used to study the behavior of $\mathrm{H}$ in $c$-Si [17] and $a$-Si [18]. The cutoffs used for Si-Si, Si-H, and H-H interactions are 2.8, 2.3, and $3.1 \AA$, respectively.

In order to generate $c$-Si $/ a$-Si:H interface models we considered different $c$-Si surface orientations, namely: $c$-Si(100) $(1 \times 1), c$-Si(100) $(2 \times 1)$, and $c$-Si(111). $c$-Si(100) and $c$ $\mathrm{Si}(111)$ surface orientations are the most common in commercial Si wafers for fabricating electronic devices [19]. Our simulation cells were elongated in the X direction, which was taken perpendicular to the surface. Periodic boundary conditions were only applied in $\mathrm{Y}$ and $\mathrm{Z}$ directions, which resulted in two free surfaces. In the case of the simulation cells for $c$-Si $(100)(1 \times 1)$ and $c$-Si $(100)(2 \times 1), Y$ and $\mathrm{Z}$ directions were [110] and [110], respectively. The cells contained $144 \mathrm{Si}$ atoms, and surfaces were passivated with $48 \mathrm{H}$ atoms in $c-\operatorname{Si}(100)(1 \times 1)$, and $24 \mathrm{H}$ atoms in $c$ - $\mathrm{Si}(100)(2 \times 1)$. In the simulation cell for

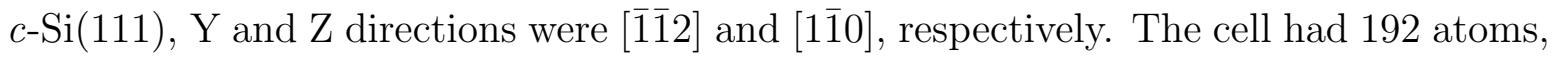


with $32 \mathrm{H}$ atoms used for passivating the surface. Our different passivated $c$-Si surfaces are represented in Fig. 1, and the total sizes and details on the number of atoms in our supercells are summarized in Table 1.

The $a$-Si:H part was generated through a quench-from-melt process. Simulation cells used had the same orientations as the corresponding $c$-Si part. They were also elongated in the $\mathrm{X}$ direction, but we applied periodic boundary conditions in the three spatial directions for the quench-from-melt process. The $a$-Si:H simulation cell used for building the $c$-Si(100)/ $a$-Si:H interface had $144 \mathrm{Si}$ atom and $15 \mathrm{H}$ atoms, while the one used for the $c$-Si(111)/a-Si:H interface had $192 \mathrm{Si}$ atom and $20 \mathrm{H}$ atoms. Initially, $\mathrm{H}$ atoms were randomly introduced in a $c$-Si simulation cell in bond-center sites, as the midpoint of $\mathrm{Si}-\mathrm{Si}$ bonds is the minimum energy position for $\mathrm{H}$ atoms in $c$-Si [17]. $\mathrm{H}$ atoms were placed with a minimum mutual separation of $4.7 \AA$ with a concentration of about $10 \%$, which is the typical $\mathrm{H}$ content in $a$-Si:H for photovoltaic applications. Once $\mathrm{H}$ atoms were introduced, the simulation cell was heated from $300 \mathrm{~K}$ to $2000 \mathrm{~K}$ with a rate of $10^{15} \mathrm{~K} / \mathrm{s}$. Then, the resulting liquid was stabilized during $50 \mathrm{ps}$, and subsequently quenched to $0 \mathrm{~K}$ with a cooling rate of $10^{13} \mathrm{~K} / \mathrm{s}$. This cooling rate lies within the typical cooling rates used in molecular dynamics simulations [20], and it is of the order of magnitude of the experimental one adopted to prepare $a$-Si using laser glazing techniques [21]. Total sizes and the number of atoms considered in our $a-\mathrm{Si}: \mathrm{H}$ simulation cells are also summarized in Table 1.

The $c$-Si $/ a$-Si:H interface was created by joining together the $c$-Si and the $a$-Si:H slabs along the $\mathrm{X}$ direction. The resulting simulation cell was relaxed using TB damped dynamics, and applying periodic boundary conditions in the three spatial directions. As a consequence, the final simulation cell had two different $c$-Si/ $a$-Si:H interfaces. The obtained configurations were used as input for density functional theory (DFT) calculations for evaluating their electronic properties, particularly at the interface. We used the plane wave DFT computational code ABINIT [22], performing an additional DFT relaxation before calculating the electronic properties. The DFT calculations were performed using Goedecker-Teter-Hutter LDA pseudopotentials [23], a $1 \times 3 \times 2 \mathrm{k}$-point mesh, and an energy cut-off of $15 \mathrm{Ha}$. We started the DFT-LDA geometry optimization from atomic positions relaxed within the TBMD scheme, rescaling atomic coordinates by the ratio of DFT-LDA vs. TB equilibrium lattice constants. The additional relaxation was performed only on the non-crystalline part of the sample, plus the first two layers of the crystalline part adjacent to the amorphous, while the central layers of the crystal were kept fixed in their ideal position. The atomic displacements turned out to decay rapidly with the minimization steps and converged within $5 \cdot 10^{-2} \AA$ at the end of the relaxation.

The sizes and the total number of atoms in our final simulation cells are shown in Table 1. The minimum separation between periodic images of the interfaces is the thickness of the thinner slab in the cell: $1.62 \mathrm{~nm}$ for $c$-Si(100) $(1 \times 1) / a-\mathrm{Si}: \mathrm{H}$ and $c$ $\mathrm{Si}(100)(2 \times 1) / a-\mathrm{Si}: \mathrm{H}$, and $1.87 \mathrm{~nm}$ for $c-\mathrm{Si}(111) / a-\mathrm{Si}: \mathrm{H}$.

We calculated the electronic density of states (EDOS) of all our relaxed 


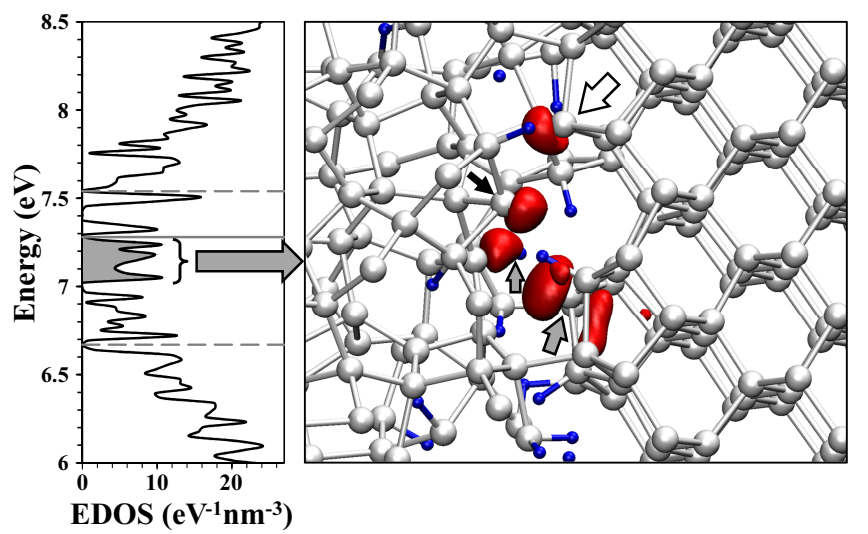

Figure 2. (Color online) (Left) EDOS of one of the $c$-Si(100) $(2 \times 1) / a$-Si:H interfaces. The shadowed region of the EDOS shows the electronic states that have been selected for analyzing their spatial localization. Horizontal gray dashed lines indicate the bulk gap region, while horizontal gray solid line indicates the position of the Fermi level. (Right) Spatial localization of the wavefunctions of the electronic states selected. Clouds represent the isosurface at $20 \%$ of the maximum of the wavefunction associated to the electronic states selected. Arrows indicate relevant atomic configurations (see Sec. 3.2 for further details).

configurations at the DFT-LDA levelł. We focused our analysis of the EDOS on energies at the band edges and at the mid-gap region. We selected different energy intervals, and we analyzed the spatial localization of the wavefunctions of the electronic states in the energy interval considered. As an example of our procedure, we show in the left side of Fig. 2 the EDOS of one of the $c$-Si $(100)(2 \times 1) / a$-Si:H interfaces. We indicate in grey the peaks associated to the analyzed electronic states, which lay in the gap region in this particular example. The right side of Fig. 2 shows the spatial localization of the wavefunction of the selected electronic states, and the atomic structures responsible form them. A detailed description of the atomic structures found following this procedure will be given in Sec. 3.2.

\section{Results and discussion}

\subsection{Structural properties}

We found that $\mathrm{H}_{2}$ molecules are formed at the $c$-Si(100)(1×1)/a-Si:H interfaces after the atomic relaxation, as indicated by empty arrows in Figs. 3 (a) and (b). The bonding distance of $\mathrm{H}$ atoms in the molecules is $0.81 \AA$, in agreement with previous ab initio studies of $\mathrm{H}$ in $c$-Si [24]. According to the size of our simulation cells, the average number of $\mathrm{H}_{2}$ molecules found is 0.4 per $\mathrm{nm}^{2}$. Interesting enough, no $\mathrm{H}_{2}$ molecules were

$\ddagger$ Such an approach for the study of the electronic structure clearly represents a compromise, allowing us to work at a paremeter-free, ab-initio level, while keeping the computational cost under control. A more refined theoretical approach, such as e.g. a GW calculation, would be numerically unaffordable due to the large simulation cells and multiple configurations considered, and would be beyond the scope of the present work. 


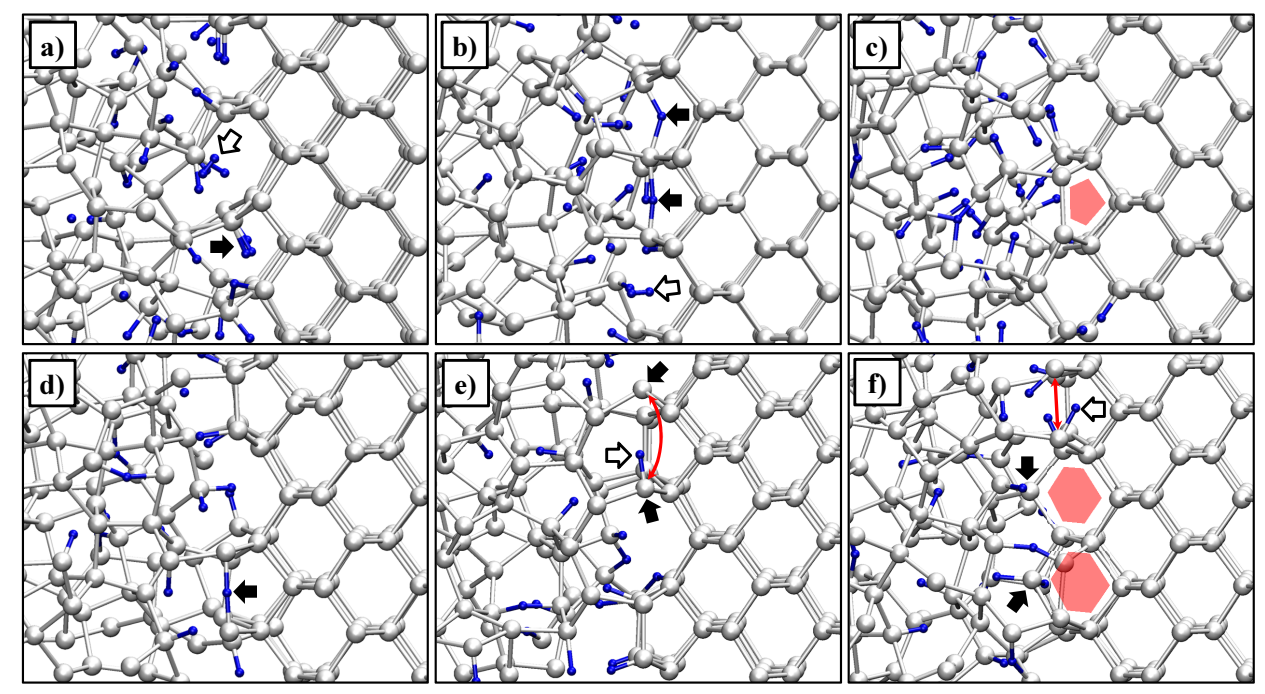

Figure 3. (Color online) Representative atomic rearrangements observed at $c$ $\mathrm{Si}(100)(1 \times 1) / a-\mathrm{Si}: \mathrm{H}(\mathrm{a}, \mathrm{b}, \mathrm{c})$ and $c$-Si $(100)(2 \times 1) / a$-Si:H interfaces (d, e, f). (a) Formation of an $\mathrm{H}_{2}$ molecule (indicated by the empty arrow) and reorganization of the remaining Si-H bonds (indicated by the black arrow) towards a neighboring Si atom at the crystalline side of the interface. (b) Formation of an $\mathrm{H}_{2}$ molecule (indicated by the empty arrow) and $\mathrm{H}$ bridges (indicated by solid arrows). (c) Complete $2 \times 1$ reconstruction. The typical pentagon of the $2 \times 1$ reconstruction is indicated by the shaded (red) area to guide the eye. (d) $\mathrm{H}$ bridge formed in a $2 \times 1$ reconstruction. Degradation of the $2 \times 1$ reconstruction by the inclusion of $\mathrm{H}$ atoms (empty arrow at the upper part of panels (e) and (f)), or by the interaction with Si atoms of the amorphous side (solid arrows of panel (f)) resulting in the hexagon typical of $c$-Si, indicated in panel (f) by the shaded (red) area.

found in the other interface models. This fact is a consequence of the larger $\mathrm{H}$ atomic surface density of the $c$-Si $(100)(1 \times 1) / a$-Si:H interface than in the other interfaces here considered, as it appears from Table 1 . In addition to the generation of $\mathrm{H}_{2}$ molecules, we observed other relevant atomic rearrangements in the $c$-Si $(100)(1 \times 1) / a$-Si:H interfaces, which are also summarized in Fig. 3, and quantified in Table 2.

In some cases, the formation of the $\mathrm{H}_{2}$ molecule at the $c$-Si $(100)(1 \times 1) / a$-Si:H interface leave some $\mathrm{Si}$ atoms at the crystalline side bonded only to one $\mathrm{H}$ atom. The remaining Si-H bond is redirected towards a neighboring $\mathrm{Si}$ atom of the crystalline side of the interface, as it is indicated by the solid arrow in Fig. 3 (a). The Si-H bond that remains after the formation of the $\mathrm{H}_{2}$ molecule can also connect two neighboring $\mathrm{Si}$ atoms at the crystalline side of the interface forming a $\mathrm{H}$ bridge, as it is shown in Fig. 3 (b) by solid arrows. This process can also end in the formation of a Si-Si bond between two neighboring $\mathrm{Si}$ atoms at the $c$-Si $(100)(1 \times 1) / a-\mathrm{Si}: \mathrm{H}$ interface provided that the $\mathrm{H}$ atom of the $\mathrm{H}$ bridge is missing, as it is representatively shown in Fig. 3 (c). The additional Si-Si bond contributes to the formation of the typical pentagon present at the $2 \times 1$ termination, indicated by the shaded (red) pentagon in Fig. 3 (c). The initial $1 \times 1$ termination of the crystalline side of the interface has been so transformed into the $2 \times 1$ termination. As previously mentioned, $\mathrm{H}$ atoms tend to occupy the central position of 


\begin{tabular}{ll}
\hline $\boldsymbol{c}$-Si $(\mathbf{1 0 0})(\mathbf{1} \times \mathbf{1}) / \boldsymbol{a}$-Si:H & \\
Rearrangements & $50 \%$ \\
H bridges & $10 \%$ \\
Pentagons & $15 \%$ \\
Hexagons & $19 \%$ \\
Deviated Si-H bonds & $56 \%$ \\
\hline $\boldsymbol{c}$-Si $(\mathbf{1 0 0})(\mathbf{2} \times \mathbf{1}) / \boldsymbol{a}$-Si:H & \\
Si-Si dimers modified & $21 \%$ \\
H bridges & $20 \%$ \\
Broken dimers & $50 \%$ \\
Hexagons & $30 \%$ \\
\hline $\boldsymbol{c}$-Si $(\mathbf{1 1 1}) / \boldsymbol{a}$-Si:H & \\
Si-H bonds & $33 \%$ \\
Si-Si bonds & $67 \%$ \\
\hline
\end{tabular}

Table 2. Summary of the atomic rearrangements observed in the different interface models considered (see Sec. 3.1 for details).

Si-Si bonds [17]. Thus, H bridges formed at the $c$-Si $(100)(1 \times 1) / a$-Si:H interface seems to be an intermediate step in the transformation from the $1 \times 1$ termination to the $2 \times 1$ termination once the $\mathrm{H}$ atom of the bridge escapes.

The simulation cells considered for modeling the $c$-Si(100) $(1 \times 1) / a$-Si:H interface have $24 \mathrm{Si}$ atoms at the $c$-Si $(100)(1 \times 1)$ termination, each of them initially bonded to 2 $\mathrm{H}$ atoms (which gives the $48 \mathrm{H}$ atoms at the crystalline side, as reported in Table 1). We found that on average $50 \%$ of these Si atoms rearranged in one of the structures discussed in the previous paragraph. $56 \%$ of these rearrangements resulted in the deviation of $\mathrm{H}$ atoms towards a neighboring Si atom of crystalline side of the interface (black arrow of Fig. $3(\mathrm{a})), 19 \%$ in the formation of an hexagon, $15 \%$ in the formation of a Si-Si dimer, and $10 \%$ in the formation of an $\mathrm{H}$ bridge.

The number of atomic rearrangements observed at $c$-Si $(100)(2 \times 1) / a$-Si:H interfaces was smaller than at $c$-Si $(100)(1 \times 1) / a-\mathrm{Si}: \mathrm{H}$ interfaces due to the higher stability of $c$-Si(100)(2×1) termination: on average $79 \%$ of the Si-Si dimers did not suffer any modification, except for small atomic displacements. In free standing $c$-Si without $\mathrm{H}$ passivation, Si atoms bond to 3 neighboring Si atoms at crystalline lattice positions in the $c$-Si $(100)(2 \times 1)$ termination, while they only bond to 2 neighboring $\mathrm{Si}$ atoms in the $c$-Si $(100)(1 \times 1)$ termination. Therefore atoms have a weaker crystalline reference at $c$-Si $(100)(1 \times 1) / a$-Si:H interfaces. Nevertheless, we could observe some relevant atomic rearrangements that are related to those observed in $c$-Si $(100)(1 \times 1) / a$-Si:H interfaces. In Fig. 3 (d) it can be seen how a $\mathrm{H}$ bridge has been formed by the movement of a $\mathrm{H}$ atom into a $2 \times 1 \mathrm{Si}-\mathrm{Si}$ dimer at the interface (indicated by the solid arrow). If more $\mathrm{H}$ atoms move towards the $2 \times 1 \mathrm{Si}$-Si dimers, dimers can be degraded as shown in the upper part of Figs. 3 (e) and (f): the empty arrow indicates the $\mathrm{H}$ atom that breaks the Si-Si dimer, 
which initially formed by the Si atoms marked with solid arrows. We also observed the formation of hexagons at the $c-\mathrm{Si}(100)(2 \times 1) / a$-Si:H interface due to the interaction of $\mathrm{Si}$ atoms of the amorphous side of the interface with $2 \times 1 \mathrm{Si}-\mathrm{Si}$ dimers, as it is shown in the lower part of Fig. 3 (f). The interaction of Si atoms of the amorphous side in the $c$-Si $(100)(1 \times 1) / a$-Si:H interface can also induce the formation of these hexagons, as it can be seen at the lower part of Fig. 3 (c). From the $21 \%$ of modified Si-Si dimers, $50 \%$ of these modifications implied the rupture of the Si-Si dimer due to the interaction of $\mathrm{H}$ atoms with the Si atoms of the dimer (i.e. the degradation of the $2 \times 1$ reconstruction to the $1 \times 1$ one), $30 \%$ the formation of an hexagon, and $20 \%$ the formation of a $\mathrm{H}$ bridge.

Finally, we did not find any atomic rearrangement at $c$-Si(111) $/ a$-Si:H interfaces. This interface has a much higher stability than the others since for the Si atoms at (111) termination, only the Si-H bond can be deviated or broken in the presence of the amorphous part, but the remaining three bonds towards the bulk keep the tetrahedral bonding configuration of $c$-Si and tie the atom to its position at the interface. On average, we found that $67 \%$ of the initial $\mathrm{Si}-\mathrm{H}$ bonds of the $c$-Si(111) termination turn into Si-Si bonds after interacting with the amorphous side.

The substrate orientation dependence of the light induced degradation of $c$-Si/a$\mathrm{Si}: H$ has been linked to the larger probability of atomic rearrangements at $c-\mathrm{Si}(100) / a$ $\mathrm{Si}: H$ than at $c-\mathrm{Si}(111) / a-\mathrm{Si}: \mathrm{H}$ [8]. In particular, the mechanism proposed for the degradation of the interface was the Si-Si dimer break up [25]. The results obtained in the present atomistic study can help to shed some light into this process, in combination with experiments of $\mathrm{H}$ effusion. Experiments show that annealing treatments of $c$-Si/ $a$ $\mathrm{Si}: \mathrm{H}$ interfaces result in $\mathrm{H}_{2}$ effusion $[6,26]$. The effusion in $c-\mathrm{Si} / a-\mathrm{Si}: \mathrm{H}$ can be interpreted on the basis of the results of thermal desorption experiments on H-passivated $c$-Si. H desorption presents a high temperature peak associated to monohydride terminations of $c$-Si (i.e. one $\mathrm{H}$ atom per $\mathrm{Si}$ atom at the surface), and a low temperature peak associated to higher hydrides [8]. In the case of $c$-Si(100), both peaks have the same intensity, which is related to a transformation of the $c$ - $\mathrm{Si}(100)(1 \times 1)$ termination to a $c$ $\operatorname{Si}(100)(2 \times 1)$ termination with temperature. This transformation modifies the dihydride termination of the $c$-Si $(100)(1 \times 1)$ surface, resulting in the low peak temperature, while the high peak temperature is due to the degradation of the monohydride termination of the $c$-Si $(100)(2 \times 1)$ surface. In the case of $c$-Si(111) the high temperature peak is much more intense [8], as a consequence of the monohydride termination of this interface. As the peak associated to dihydride terminations occurs at low temperature, it indicates that $\mathrm{Si}-\mathrm{H}$ bonds are easily broken for dihydride terminations.

We have also found a larger number of atomic rearrangements at the $c$ $\mathrm{Si}(100)(1 \times 1) / a-\mathrm{Si}: \mathrm{H}$ interface than at the $c$-Si $(100)(2 \times 1) / a$-Si:H one: while $50 \%$ of the $\mathrm{Si}$ atoms of the $c$-Si $(100)(1 \times 1)$ termination suffered large rearrangements at the $c$-Si(100) $(1 \times 1) / a$-Si:H interface, only $21 \%$ of $\mathrm{Si}$-Si dimers were modified at the $c$ $\operatorname{Si}(100)(2 \times 1) / a$-Si:H interface. From these rearrangements, $15 \%$ of them resulted in the $2 \times 1$ reconstruction in the $c$-Si $(100)(1 \times 1) / a$-Si:H interface, while $80 \%$ implied the complete rupture of $\mathrm{Si}-\mathrm{Si}$ dimers in the $c$-Si(100) $(1 \times 1) / a$-Si:H interface $(50 \%$ of 


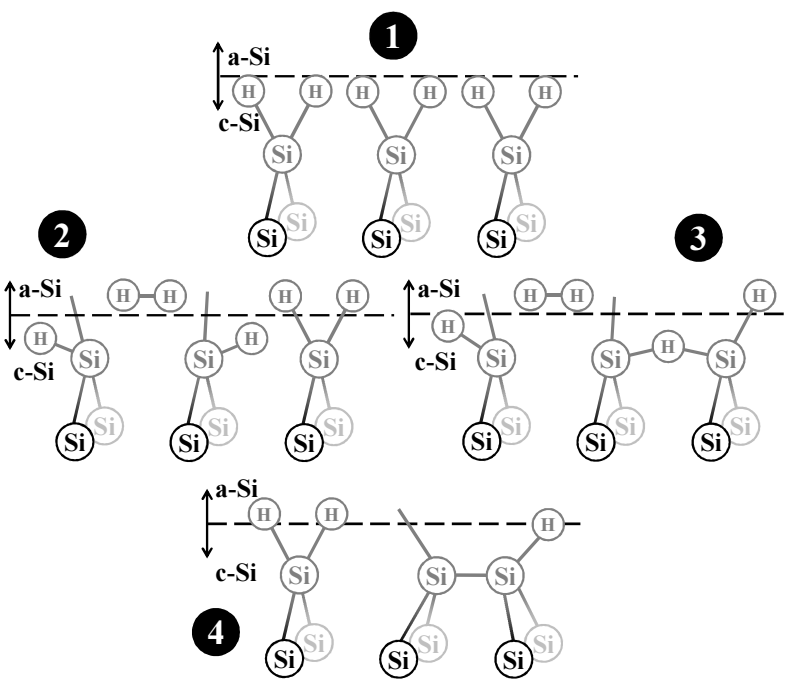

Figure 4. Schematic representation of the atomic rearrangements at $c-\mathrm{Si}(100) / a-\mathrm{Si}: \mathrm{H}$ interfaces. $\mathrm{H}_{2}$ molecules can be formed at the $1 \times 1$ terminated interface, and lead to the restructuration of Si-H bonds $(1 \rightarrow 2)$ or to the formation of $\mathrm{H}$ bridges $(1 \rightarrow 3)$. Further rearrangements, such as the removal of the $\mathrm{H}$ atom from the bridge or the interaction of the dangling bonds after the formation of the $\mathrm{H}_{2}$ molecule, can result in the formation of a Si-Si dimer at the interface $(2,3 \rightarrow 4)$. A Si-Si dimer can also be broken due to the interaction with $\mathrm{H}$ atoms or with $\mathrm{Si}$ atoms of the amorphous side $(4 \rightarrow 3)$. The gray scale schematically represents depth and is to guide the eye. Bonds towards the amorphous matrix have not been completely shown to indicate that they can bond a $\mathrm{Si}$ atom, a $\mathrm{H}$ atom, or they can be missing.

$\mathrm{Si}-\mathrm{Si}$ dimer rupture by $\mathrm{H}$ interaction plus $30 \%$ of hexagon formation). This gives a total percentage of $50 \% \cdot 15 \%=7.5 \%$ for the $(1 \times 1) \rightarrow(2 \times 1)$ transformation, and $21 \% \cdot 80 \%=16.8 \%$ for the $(2 \times 1) \rightarrow(1 \times 1)$ transformation, which indicates that the latter process is more probable. With respect to $\mathrm{H}$ bridges, their total percentage is $5 \%$ and $4.2 \%$ in the $c$-Si $(100)(1 \times 1) / a$-Si:H and the $c$-Si $(100)(2 \times 1) / a$-Si:H interfaces, respectively. The early model proposed by Carlson [25] described the degradation of the $2 \times 1$ termination from the interaction of $\mathrm{H}$ atoms with the Si atoms of the dimer. Our findings support this hypothesis, and also points out additional degradation mechanisms of $2 \times 1$ Si-Si dimers due to the interaction with the amorphous matrix. These processes have been schematically represented in Fig. 4.

\subsection{Electronic properties}

The next step of our study is the identification of the relevant atomic structures that result in electronic states localized at $c$-Si/a-Si:H interfaces. For this purpose, we followed the procedure described at the end of Sec. 2: we analyzed the spatial localization of the wavefunctions of the electronic states lying within the gap region. In the particular case of Fig. 2 we could correlate the mid gap states shadowed in EDOS (left side of Fig. 2) to the structures responsible for them (right side of Fig. 2), which are clearly characterized by dangling bonds. The dangling bond indicated by the upper empty 

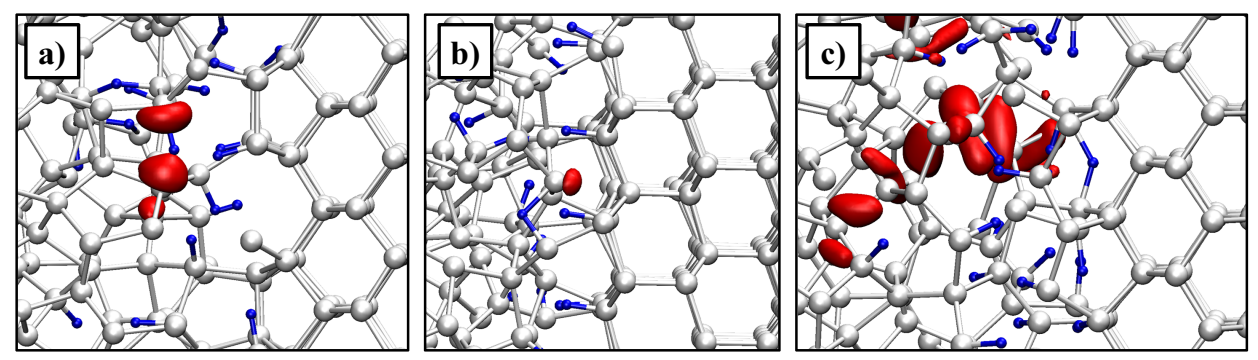

Figure 5. (Color online) Atomic configurations of the atomic structures associated to levels in the gap region of the analyzed samples: (a) H bridges, (b) dangling bonds, and (c) strained bonds. Red clouds represent the isosurface at $20 \%$ of the maximum of the wavefunction associated to each defect.

\begin{tabular}{lccc}
\hline & H bridge & Dangling bond & Strained bond \\
\hline$c-\mathrm{Si}(100)(1 \times 1) / a-\mathrm{Si}: \mathrm{H}$ & $58 \%$ & $24 \%$ & $18 \%$ \\
$c-\mathrm{Si}(100)(2 \times 1) / a-\mathrm{Si}: \mathrm{H}$ & $44 \%$ & $49 \%$ & $7 \%$ \\
$c-\mathrm{Si}(111) / a-\mathrm{Si}: \mathrm{H}$ & $29 \%$ & $59 \%$ & $12 \%$ \\
\hline
\end{tabular}

Table 3. Percentage of defects found in the vicinity of the interfaces for the different interface models considered.

arrow is due to the absence of the $\mathrm{H}$ bond that initially passivated the $2 \times 1 \mathrm{Si}-\mathrm{Si}$ dimer at the crystalline side of the interface. There is another dangling bond at a different $2 \times 1 \mathrm{Si}-\mathrm{Si}$ dimer that is indicated by the lower gray arrow. In this case the $\mathrm{H}$ atom of the $2 \times 1$ termination has moved to the amorphous side forming a $\mathrm{Si}-\mathrm{H}$ bond (indicated by the smaller gray arrow), leaving behind the dangling bond. The generation of the dangling bond also induces some electronic localization around the $\mathrm{Si}-\mathrm{H}$ bond formed at the amorphous side of the interface. Finally, there is another dangling bond in the amorphous side of the interface, indicated by the black solid arrow. This dangling bond is within the space between two consecutive $2 \times 1 \mathrm{Si}$-Si dimers at the crystalline side of the interface.

Similarly, this procedure allowed us to identify the atomic structures at the $c$ $\mathrm{Si} / a$-Si:H interfaces responsible for the energy levels observed in the gap region of the EDOS. We could classify the observed atomic structures in the following categories: dangling bonds, strained bonds, and $\mathrm{H}$ bridges. We have shown in Fig. 5 a representative example of these atomic structures, along with the spatial localization of the associated wavefunction. Dangling bonds have a characteristic electronic state spatially localized at the dangling bond itself (i.e. at the place where the bond is missing). We also found regions of strained bonds, which have a very characteristic shape of the associated electronic cloud [27, 28]: cylindrical-like clouds crossing Si-Si bonds. Finally, H bridges had the same structure as those observed at the $c$-Si(100)/ $a$-Si:H interface: a $\mathrm{H}$ atom connecting two Si atoms. The electronic clouds associated to this structure are at both sides of the $\mathrm{H}$ atom, and behind the $\mathrm{Si}$ atoms that form the $\mathrm{H}$ bridge.

In Table 3 we summarize the percentage of defects found in the vicinity of the interfaces for the different interface models considered. The most abundant defect is the 


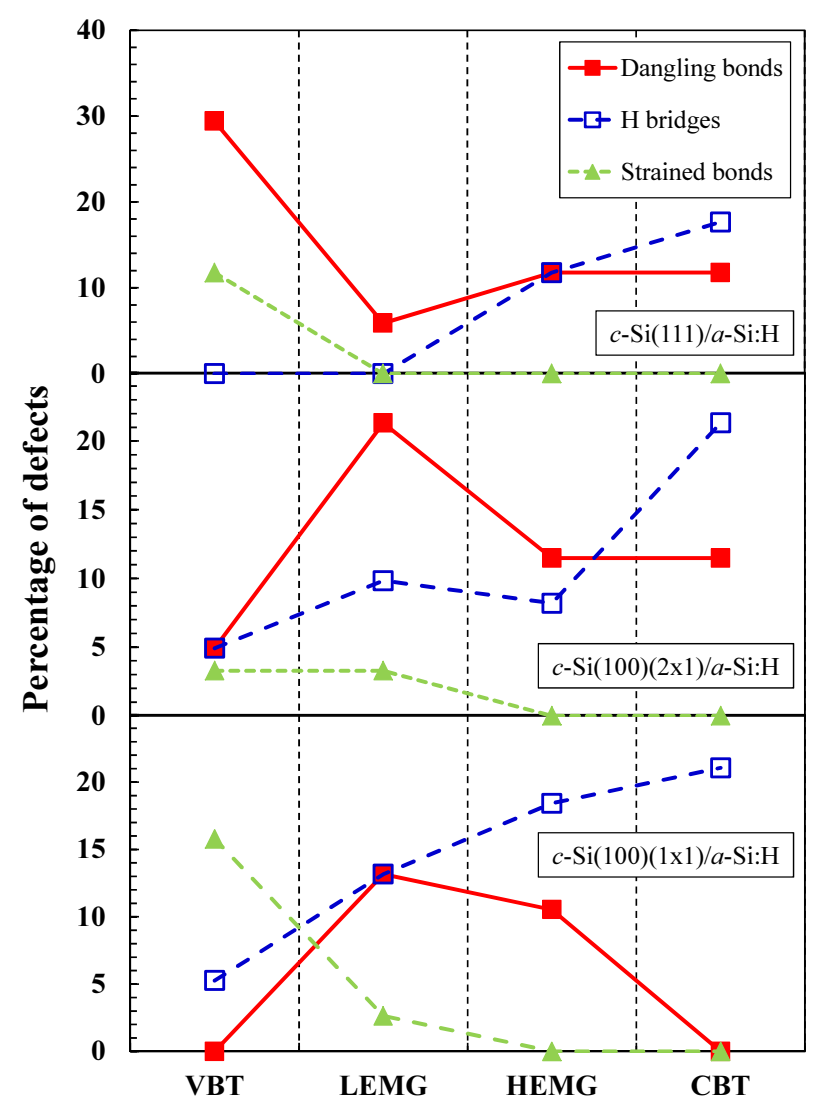

Figure 6. Percentage of defects observed at the vicinity of the interface for the different interface models considered as a function of the region of the gap were they appear: valence band tail (VBT), low-energy mid-gap (LEMG), high-energy mid-gap (HEMG), and conduction band tail (CBT).

$\mathrm{H}$ bridge at the $c-\mathrm{Si}(100)(1 \times 1) / a$-Si:H interface, due to the higher $\mathrm{H}$ surface density at this interface. In the case of $c$-Si $(100)(2 \times 1) / a$-Si:H and $c$-Si $(111)(1 \times 1) / a$-Si:H interfaces, dangling bonds are the predominant defect. In these interfaces, Si atoms at the crystalline side of the interface have 3 bonds with Si atoms at the crystalline side, and the remaining bond is formed with the $\mathrm{H}$ atom which is initially towards the amorphous side. Since this is the weaker bond, it can be easily broken and thus a dangling bond is created. The relevance of strained bonds is higher at $c$-Si $(100)(1 \times 1) / a$-Si:H. At this interface, $\mathrm{Si}$ atoms at the crystalline side of the interface have only two bonds to $\mathrm{Si}$ atoms of the crystalline side. Therefore they are not as tied as in the other interfaces and they can rearrange easily when interacting with the amorphous side, and thus induce observed the strained bonds.

In Fig. 6 we represent how the observed defects at the vicinity of the interfaces are distributed along the gap region. Despite the variety of percentages shown in Fig. 6 , a general trend can be extracted relating each of the observed atomic structures to the region around the gap where the corresponding electronic state lays. This trend is schematically summarized in Fig. 7. States associated to dangling bonds present a strong nearby spatial localization. The energy levels fall within the mid gap region, thus 


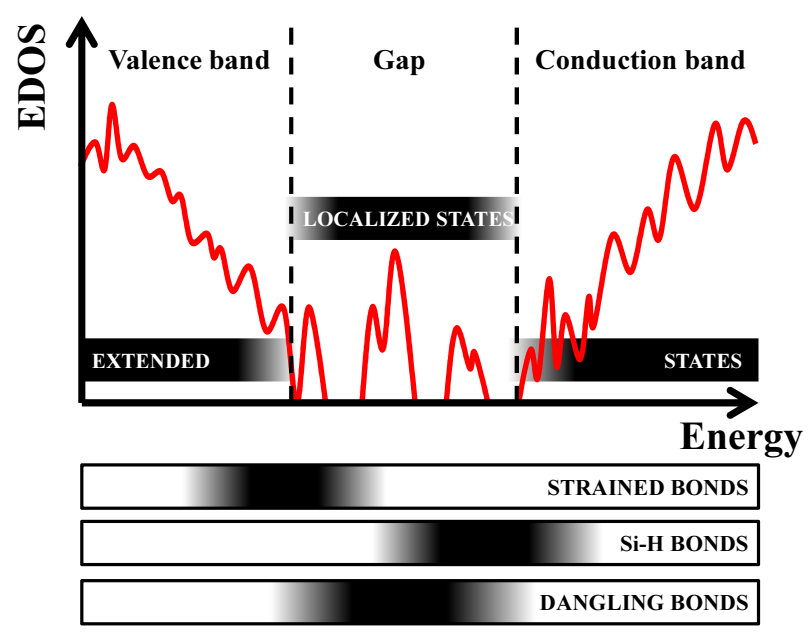

Figure 7. Schematic representation of the region in the EDOS where the observed defects usually appear.

providing the well known amphoteric behavior of dangling bonds [9]. Strained bonds induce in turn states around the valence band edge (valence band tail and low-energy part of the mid gap). These states are not as localized as those of dangling bonds, rather they can encompass several atoms and act as hole traps [28, 29], which agrees with the region of the gap where they appear. Finally, $\mathrm{H}$ bridges also present a high spatial localization around the $\mathrm{Si}-\mathrm{H}-\mathrm{Si}$ atoms forming the bridge, and the associated defect levels appear in the upper half region of the gap and at the conduction band edge. According to the energy region where these levels appear, they can mainly interact with the electrons at the interface.

The breaking of Si-H bonds and the generation of dangling bonds are commonly used to explain the electrical properties of c-Si/a-Si:H heterojunctions $[6,8]$. Furthermore, recent studies have probed the presence of dangling bonds in the c$\mathrm{Si}(111)$ /a-Si:H interface [30]. Nevertheless, strained bonds have not been yet considered in the models describing phenomena nearby $c$-Si/ $a-\mathrm{Si}: \mathrm{H}$ interfaces. It has been recently found that the density of hole traps in $a-\mathrm{Si}: \mathrm{H}$ generated by plasma-enhanced chemical vapor deposition on $c$-Si can be significantly reduced under prolonged light illumination, and subsequent thermal annealing can restore the initial density of hole trap states in the sample to its original value before illumination [31]. These facts resemble the experimental results of light induced degradation of $c$-Si/a-Si:H interfaces [8]. As previously mentioned, atomistic simulations found that states associated to strained bonds are at valence band tails and can act as hole traps in $a$-Si $[28,29]$. This localization induced by strained bonds can result in quantum confinement in $c$-Si nanocrystals embedded in $a$-Si:H [32]. Furthermore, light illumination can induce the generation and recombination of the states associated to strained bonds [27], being the atomic rearrangement proposed for the generation/recombination very similar to the bond switch mechanism observed in bulk $a$-Si for the self-diffusion under annealing [33]. Thus, the observed variations of the density of hole traps by illumination and annealing in 
bulk $a$-Si:H might be related to states associated to strained bonds, and their role in the phenomena occurring at $c-\mathrm{Si} / a-\mathrm{Si}: \mathrm{H}$ interfaces can be as important as the role of dangling bonds.

\section{Conclusions}

We have used atomistic simulations to study the structural and electronic features of $c$ $\mathrm{Si} / a$-Si:H interfaces for different orientations and terminations of the $c$-Si substrate. We have analyzed first how $\mathrm{H}$ and $\mathrm{Si}$ atoms interact at the interface, extracting the relevant steps that lead to the transformation from $c$-Si(100) $(1 \times 1) / a$-Si:H to $c$-Si $(100)(2 \times 1) / a$ $\mathrm{Si}: \mathrm{H}$ observed in the experiments. The atomic rearrangements found are more common at the $c$-Si $(100)(1 \times 1) / a$-Si:H interface than at the $c-\mathrm{Si}(100)(2 \times 1) / a-\mathrm{Si}: \mathrm{H}$ one. The observed interactions between $\mathrm{Si}$ and $\mathrm{H}$ atoms complement the early model developed by Carlson [25]. We did not find large atomic rearrangements at $c-\mathrm{Si}(111) / a-\mathrm{Si}: \mathrm{H}$ interfaces. We only found deviations or break ups of Si-H bonds, but without affecting the Si atoms of the crystalline side of the interface.

We have also identified the relevant atomic configurations that result in the defect states at $c$-Si $/ a$-Si:H interfaces, namely dangling bonds, $\mathrm{Si}-\mathrm{H}$ bonds, and strained bonds, showing that they show up mainly in the central, upper, and lower part of the energy gap, respectively. While dangling bonds and $\mathrm{Si}-\mathrm{H}$ bonds have been commonly used for describing the defect states at $c$-Si/ $a$-Si:H interfaces, we have found that states associated to strained bonds are also present at the vicinity of $c$-Si/a-Si:H interfaces. Previous studies found that these states are very related to trapping centers of charge carriers and light induced defects in bulk $a$-Si, which indicate that they can also have an important role in the physics of $c-\mathrm{Si} / a-\mathrm{Si}: \mathrm{H}$ interfaces.

\section{Acknowledgments}

We would like to thank M. Palummo for useful discussion. This work was partially funded by Spanish DGI under project number TEC2011-27701 (I.S.), and supported by the European Union through the ETSF e-I3 Grant No. INFRA-2007-211956 (M.C., G.O.), and by the ETSF [34] under projects number 366 and 469 (G.O., I.S.). Computing time has been provided by the CINECA, through the Italian SuperComputing Resource Allocation - ISCRA initiative.

\section{References}

[1] Y. Tsunomura et al. Sol. Energ. Mater. Sol. Cells, 93:670, 2009.

[2] T. Mishima, M. Taguchi, H. Sakata, and E. Maruyama. Sol. Energ. Mater. Sol. Cells, 95:18, 2011.

[3] S. Tohoda et al. J. Non-Cryst. Solids, 358:2219, 2012.

[4] T. F. Schulze, H. N. Beushausen, T. Hansmann, L. Korte, and B. Rech. Appl. Phys. Lett., 95:182108, 2009. 
[5] A. Descoeudres, L. Barraud, S. De Wolf, B. Strahm, D. Lachenal, C. Guérin, Z. C. Holman, F. Zicarelli, B. Demaurex, J. Seif, J. Holovsky, and C. Ballif. Appl. Phys. Lett., 99:123506, 2011.

[6] S. De Wolf and M. Kondo. J. Appl. Phys., 105:103707, 2009.

[7] H. Plagwitz, B. Terheiden, and R. Brendel. J. Appl. Phys., 103:094506, 2008.

[8] S. De Wolf, B. Demaurex, A. Descoeudres, and C. Ballif. Phys. Rev. B, 83:233301, 2011.

[9] S. Olibet, E. Vallat-Sauvain, and C. Ballif. Phys. Rev. B, 76:035326, 2007.

[10] L. Colombo. Riv. Nuovo Cimento, 28(10):1, 2005.

[11] L. Goodwin, A. J. Skinner, and D. G. Pettifor. EPL (Europhysics Letters), 9:701, 1989.

[12] C. Z. Wang, C. T. Chan, and K. M. Ho. Phys. Rev. Lett., 66:189, 1991.

[13] E. G. Song, E. Kim, Y. H. Lee, and Y. G. Hwang. Phys. Rev. B, 48:1486, 1993.

[14] M. T. Zawadzki, W. Luo, and P. Clancy. Phys. Rev. B, 63:205205, 2001.

[15] G. Servalli and L. Colombo. EPL (Europhysics Letters), 22:107, 1993.

[16] Eunja K. and Young Hee L. Phys. Rev. B, 49:1743, 1994.

[17] G. Panzarini and L. Colombo. Phys. Rev. Lett., 73:1636, 1994.

[18] S. Lanzavecchia and L. Colombo. EPL (Europhysics Letters), 36:295, 1996.

[19] W. C. O'Mara, R. B. Herring, and L. P. Hunt. Handbook of semiconductor silicon technology. Noyes Publications, Park Ridge, New Jersey, U.S.A., 1990.

[20] M. Ishimaru, S. Munetoh, and T. Motooka. Phys. Rev. B, 56:15133, 1997.

[21] R. Zallen. The Physics of Amorphous Solids (Willey, New York, 1983).

[22] X. Gonze et al. Computer Phys. Commun., 180:2582, 2009. http://www.abinit.org.

[23] S. Goedecker, M. Teter, and J. Hutter. Phys. Rev. B, 54:1703, 1996.

[24] Y. Okamoto, M. Saito, and A. Oshiyama. Phys. Rev. B, 56:R10016, 1997.

[25] D.E. Carlson. Applied Physics A, 41:305, 1986.

[26] S. De Wolf and M. Kondo. Appl. Phys. Lett., 91:112109, 2007.

[27] L. K. Wagner and J. C. Grossman. Phys. Rev. Lett., 101:265501, 2008.

[28] I. Santos, P. Castrillo, W. Windl, D. A. Drabold, L. Pelaz, and L. A. Marqués. Phys. Rev. B, 81:033203, 2010.

[29] P. A. Khomyakov, Wanda Andreoni, N. D. Afify, and Alessandro Curioni. Phys. Rev. Lett., 107:255502, 2011.

[30] B. M. George, J. Behrends, A. Schnegg, T. F. Schulze, M. Fehr, L. Korte, B. Rech, K. Lips, M. Rohrmüller, E. Rauls, W. G. Schmidt, and U. Gerstmann. Phys. Rev. Lett., 110:136803, 2013.

[31] I. Sakata, T. Kamei, and M. Yamanaka. J. Non-Cryst. Solids, 358:2048, 2012.

[32] L. Bagolini, A. Mattoni, G. Fugallo, L. Colombo, E. Poliani, S. Sanguinetti, and E. Grilli. Phys. Rev. Lett., 104:176803, 2010.

[33] I. Santos, L. A. Marqués, L. Pelaz, and L. Colombo. Phys. Rev. B, 83:153201, 2011.

[34] A.Y. Matsuura, N Thrupp, X. Gonze, Y. Pouillon, G. Bruant, and G. Onida. Comput. Sci. Eng., 14:22, 2012. http://www.etsf.eu. 